\title{
Emergency department junior medical staff's knowledge, skills and confidence with psychiatric patients: a survey
}

\author{
Jacqueline Therese Gordon ${ }^{1}$
}

The Psychiatrist (2012), 36, 186-188, doi: 10.1192/pb.bp.111.035188

${ }^{1}$ Worthing Hospital, Worthing, UK Correspondence to Jacqueline Therese Gordon(jackie.gordon@ sussexpartnership.nhs.uk)

First received 6 May 2011, revised 5 Oct 2011, accepted 9 Nov 2011

\begin{abstract}
Aims and method To determine knowledge, skills and confidence of junior medical emergency department staff in managing mental health patients. Over a 2-year period new emergency department junior doctors were given a questionnaire to complete early on in their post and prior to any mental health training. The questionnaire asked about knowledge, confidence, concerns and skills in the management of mental health patients in accident and emergency services.
\end{abstract}

Results More than half of the 32 doctors surveyed said they lacked knowledge, skills and confidence when assessing mental health patients.

Clinical implications The survey has demonstrated a need for mental health training of new doctors working in the emergency department. Effective training for such doctors can be offered by a liaison psychiatry service. This should be embedded in their teaching programme and be continually responsive to their perceived training needs.

\section{Declaration of interest None.}

Patients with mental health problems often present to the emergency department. ${ }^{1}$ Saliou et al found a $35 \%$ prevalence of psychiatric disorders in a hospital emergency service in France. $^{2}$ A London hospital emergency department reported 565 attendances of individuals with overt psychiatric problems over a 3-month period. The most frequent presenting problems were self-harm (31\%), substance misuse (mainly alcohol) (20\%), psychosis (17\%) and mood disturbance (15\%). ${ }^{3}$ Keene \& Rodriguez found that over a 3 -year period, approximately $30 \%$ of secondary care mental health patients had presented to the emergency services. ${ }^{4}$ Hisley \& Meldon report that one in six older people in an emergency department had dementia. ${ }^{5}$ Junior doctors working in this setting are likely therefore to be assessing and managing this varied patient group. The Royal College of Psychiatrists makes the following recommendations ${ }^{6}$ regarding mental health provision in accident and emergency services:

(a) emergency department personnel should have adequate knowledge of mental health issues and feel confident in making an initial assessment of people with mental health problems;

(b) staff training should include the recognition of common mental health problems and the appropriate responses as well as safety issues.

In its guidance on self-harm, the National Institute for Health and Clinical Excellence (NICE) states:

\begin{abstract}
'Mental health services and emergency department services should jointly develop regular training programmes in the psychosocial assessment and early management of self-harm, to be undertaken by all healthcare professionals who may assess or treat people who have self-harmed' (p. 51). ${ }^{7}$
\end{abstract}

In our hospital, the mental health liaison service provides two sessions of psychiatry training to new doctors near the beginning of their emergency department placement and on a rolling basis. This survey was conducted to gauge knowledge and confidence in this area before training had been received, and to inform the content of future teaching sessions. We could find no similar survey conducted previously.

\section{Method}

Between 2008 and 2010, all junior doctors who attended a psychiatry teaching session near the beginning of their emergency department placement were asked to fill out a questionnaire. All $32(100 \%)$ doctors in attendance (of a total of 36 doctors) agreed to take part in this survey and completed the questionnaire. These were foundation year 2 doctors, general practitioners, emergency care core trainees and acute care common stem trainees. J.T.G. administered the survey in person and collected it immediately after completion. Four doctors who were on leave did not take part in the training. The survey asked about the doctors' confidence, knowledge, skills and concerns when assessing 
patients with mental health problems, and how they would assess a patient who presented with self-harm.

\section{Results}

\section{Quantitative analysis}

The breakdown of responses according to number is presented in Table 1 .

1. 'Have you received any postgraduate mental health training? Out of the 32 doctors surveyed, 28 had received no psychiatry training after qualifying from medical school.

\section{2. 'Are you confident about seeing psychiatric patients in} casualty?'

Nine doctors said they were not confident about seeing psychiatric patients and none of these had had post-medical school experience in mental health. Of the nine doctors who said they were confident about assessing psychiatric patients, only three had had post-medical school psychiatry exposure. Fourteen expressed partial confidence but with some reservation.

3. 'Do you have enough knowledge of mental health to see these patients in casualty?'

Six doctors said yes, but only two of them had done postgraduate psychiatry. Seventeen said they felt their knowledge was inadequate, and the remaining nine thought they had some knowledge but it was incomplete.

4. 'Do you have the necessary skills to see mental health patients in casualty?'

About a third $(n=10)$ thought they had sufficient skills and a third $(n=11)$ thought they had insufficient skills to assess patients with mental health issues. The remaining 11 were not sure.

5. 'Do you have specific concerns with this patient group?' Twenty doctors said yes, and this will be expanded on later in this paper. Only six doctors said they had no concerns, and the remainder were concerned in part.

\section{Qualitative analysis}

2. 'Are you confident about seeing psychiatric patients in casualty?'

Nine doctors expanded on their answers: three said they thought assessing individuals who overdosed was relatively straightforward and two of these cited psychosis as an area they lacked confidence in. Three said it depended on the clinical situation as to how confident they felt.

4. 'Do you have the necessary skills to see mental health patients in casualty?'

Twenty-seven answers were expanded upon. Six respondents said they required teaching in 'acute psychosis and sedation'; eight wanted to know how to assess risk and two of these specified 'suicide' risk. Three required training in the use of the Mental Health Act 1983 and five wanted to be better able to decide whether to admit or discharge a patient with mental health issues from the emergency
Table 1 Perceived competence in assessment of mental health patients $(n=32)$

\begin{tabular}{lccc} 
& Yes & No & Partial \\
\hline 1 Postgraduate mental health training & 4 & 28 & - \\
\hline 2 Confident about seeing psychiatric & 9 & 9 & 14 \\
patients in casualty & 6 & 17 & 9 \\
\hline $\begin{array}{l}3 \text { Sufficient knowledge about mental } \\
\text { health for this post }\end{array}$ & 6 & 11 & 11 \\
\hline $\begin{array}{l}4 \text { Sufficient skills in managing mental } \\
\text { health patients }\end{array}$ & 10 & & \\
\hline $\begin{array}{l}5 \text { Concerns about assessing mental } \\
\text { health patients }\end{array}$ & 20 & 6 & 6
\end{tabular}

department. Six doctors wanted to know how to ask the right questions and take good history, and a further two wanted to learn communication/consultation skills.

\section{5. 'Do you have specific concerns with this patient group?'} Fifteen answers were expanded upon. Although these responses in part resembled expressed training needs, a number of separate concerns were expressed: those referred to managing patients who absconded from the emergency department; those who repeatedly self-harmed; those who refused to stay for psychiatric assessment; the doctor's 'inexpert' input increasing the possibility of the patient self-harming; and management of aggression with or without substance misuse.

6. 'Can you tell me, in brief, how you would assess a patient who has self-harmed?'

There was both space and time to expand on this answer in the questionnaire and 31 respondents took this opportunity. An adequate response would incorporate use of a triage tool (we use the SAD PERSONS score ${ }^{8}$ ) and recommendations from NICE guidelines: accident and emergency staff should offer a preliminary psychosocial assessment to include capacity, willingness to remain for further psychosocial assessment, level of distress and possible presence of mental illness. I also expected doctors to ask about suicide intent at the time of self-harm and subsequently. Overall, four responses were deemed inadequate:

(a) only the physical injury was addressed

(b) doctor did not assess but referred immediately to 'psych'

(c) doctor would use the SAD PERSONS score and if moderate then they would refer to 'psych'

(d) SAD PERSONS score alone was used.

A further 11 responses were acceptable in part but were incomplete in an important way: 8 doctors did not say they would ask about psychiatric history and 3 did not address suicide intent around the act. Sixteen methods of assessment were reasonably comprehensive and in particular focused on the intention around the act of self-harm, mental state examination and psychiatric history.

In its guidance on the assessment of self-harm, ${ }^{7} \mathrm{NICE}$ suggests that emergency department staff should carry out a 
preliminary psychosocial assessment which should include capacity, willingness to remain for further psychosocial assessment, level of distress, and possible presence of mental illness. In the survey, no doctor mentioned performing a capacity assessment.

\section{Discussion}

This survey, the first of its type, has demonstrated that there is a need for mental health training of new doctors working in the emergency department. This should take place as early on in their placement as possible.

Mental health foundation training does assess competency in the management of self-harm, but the majority of our doctors had not trained in mental health. Additionally, medical student training incorporates teaching on the assessment of psychiatric patients but it seems this particular knowledge is not well retained. In this sample, a large proportion of doctors said they lacked knowledge and confidence in the assessment of mental health patients, and they identified training needs in terms of knowledge and skills. When asked how they would assess self-harm, five were not able to do this adequately and a further eleven were deficient in some way. No respondent assessed capacity, as is recommended in NICE guidance. A limitation of this study was the small number of participants.

As a result of this study I now have two training sessions per emergency department doctor cohort. In the first session we discuss how to assess patients who have selfharmed, including a capacity assessment; how to perform a brief psychiatric history and mental state examination; and how to manage behavioural disturbance. The second session examines in more depth the capacity assessment, the Mental Health Act and ethical issues. The sessions are kept relatively open so that junior doctors can raise concerns and discuss problematic cases. Feedback from these sessions has been very positive.
I hope to conduct a follow-up survey after these training sessions to see whether these perceived deficits in skills, confidence and knowledge have been addressed. I also hope that the results may be useful to medical educationalists who are involved in the planning of mental health undergraduate and postgraduate curricula.

\section{About the author}

Jacqueline Therese Gordon is an associate specialist and honorary consultant in liaison psychiatry, Worthing Hospital, Worthing, UK.

\section{References}

1 Johnson S, Thornicroft G. Emergency psychiatric services in England and Wales. BMJ 2005; 311: 287-8.

2 Saliou V, Fichelle A, McLoughlin M, Thauvin I, Lejoyeux M. Psychiatric disorders among patients admitted to a French medical emergency service. Gen Hosp Psychiatry 2005; 27: 263-8.

3 Cassar S, Hodgkiss A, Ramirez A, Williams D. Mental health presentations to an inner-city accident and emergency department. Psychiatr Bull 2002; 26: 134-6.

4 Keene J, Rodriguez J. Are mental health problems associated with use of accident and emergency and health-related harm? Eur J Public Health 2006; 17: 387-93

5 Hisley F, Meldon S. The prevalence and documentation of impaired mental status in elderly emergency department patients. Ann Emerg Med 2002; 39: 248-53.

6 Royal College of Psychiatrists. Psychiatric Services to Accident and Emergency Departments (Council Report CR118). Royal College of Psychiatrists, 2004.

7 National Institute for Health and Clinical Excellence. Self-Harm: The Short-Term Physical and Psychological Management and Secondary Prevention of Self-Harm in Primary and Secondary Care (Clinical Guidelines (G16). NICE, 2004.

8 Patterson WM, Dohn $\mathrm{HH}$, Bird J, Patterson GA. Evaluation of suicidal patients: the SAD PERSONS scale. Psychosomatics 1983; 24: 343-5, $348-9$. 